\title{
Prospective, randomised, controlled trial comparing suture needle drainage and argon laser drainage of subretinal fluid
}

\author{
G W Aylward, G Orr, S D Schwartz, P K Leaver
}

\begin{abstract}
Aims-This study was designed to compare suture needle drainage (SND) with argon laser drainage (ALD) of subretinal fluid.

Methods-A prospective, randomised, controlled, clinical trial was carried out on 93 patients undergoing external drainage of subretinal fluid during scleral buckling surgery for rhegmatogenous retinal detachment. The incidence of successful drainage, incidence of subretinal haemorrhage, incidence of retinal incarceration, and incidence of retinal perforation were determined.

Results-The success rate was $97.9 \%$ in the ALD group and $84.8 \%$ in the SND group (difference $-13 \cdot 1 \%, 95 \%$ CI $-26.4 \%$ to $2 \cdot 0 \%$ ). The incidence of clinically significant subretinal haemorrhage was $4.3 \%$ in the ALD group compared with $28.3 \%$ in the SND group (difference $24.0 \%$, $95 \%$ CI $7 \cdot 6 \%$ to $40 \cdot 4 \%$ ). The incidence of incarceration was comparable in each group. There were no cases of retinal perforation.

Conclusions-The use of ALD was associated with a higher rate of successful SRF drainage, and a lower incidence of clinically significant subretinal haemorrhage than SND. ALD is preferred when an endolaser is available.
\end{abstract}

(Brf Ophthalmol 1995; 79: 724-727)

External drainage of subretinal fluid (SRF) during surgery for retinal detachment may be complicated by haemorrhage, incarceration of retina and/or vitreous, or retinal perforation. ${ }^{1-3}$ These events are associated with a high incidence of surgical failure ${ }^{4}$ and visual acuity may be affected, particularly by subretinal haemorrhage. ${ }^{56}$ Many techniques of drainage have been described, all of them aimed at producing safe and effective drainage of SRF. ${ }^{7-9}$ Argon laser drainage (ALD), first described by Bovino et al, ${ }^{10}$ has one of the lowest published incidences of complications, particularly haemorrhage. ${ }^{1112}$ Suture needle drainage (SND) has gained in popularity recently. It is a simple and quick method that achieves drainage without a large sclerotomy, so that the risk of incarceration is reduced. Experience with SND in a large series of patients undergoing scleral buckling surgery has recently been reported. ${ }^{13}$

Comparisons of the incidence of complications between different studies is difficult because of wide variations in the case mix and differences in the definition of haemorrhage. We therefore conducted a prospective, randomised, controlled clinical trial comparing ALD with SND in order to discover which was the safer and/or more effective method.

\section{Materials and methods}

We studied patients requiring drainage of subretinal fluid as part of scleral buckling surgery for rhegmatogenous retinal detachment. Eyes with a history of previous scleral buckling surgery were included, provided an interval of at least 6 weeks had transpired, and there was no evidence of inflammation. Patients with a history of bleeding disorders, and those on anticoagulants or non-steroidal anti-inflammatory drugs were excluded from the trial. Eyes with myopia greater than 12 dioptres were also excluded. The number of patients required to detect a threefold difference in the incidence of subretinal haemorrhage between the two groups was calculated using the formula of Fleiss. ${ }^{14}$ We assumed figures of $5.5 \%$ for the incidence of subretinal haemorrhage, ${ }^{11} 0.05$ for the alpha risk, and 0.8 for the statistical power required. The result of this calculation was a sample size of 144 patients in each arm of the trial.

A set of forms was prepared and marked either ALD or SND according to a randomisation derived from random number tables. The forms were placed in sealed envelopes and opened sequentially when the type of operation and the surgeon had been decided. A drainage site was chosen overlying detached retina, preferably just above or below the horizontal recti. Transillumination was not employed in selecting the drainage site. The configuration of the detachment and the site of drainage were recorded. Drainage was carried out before cryotherapy in all instances. All operations were carried out under general anaesthesia. The operating surgeon was either a consultant, vitreoretinal fellow, or senior registrar operating under supervision. In all cases the surgeon had previous experience of both techniques.

ALD was performed by first making a small $(2 \mathrm{~mm})$ radial sclerotomy. Specially designed drainage forceps ${ }^{15}$ were then used to spread the lips of the sclerotomy which was further dissected to expose a knuckle of choroid. An endolaser probe was then placed adjacent to the exposed choroid. The laser was set to argon green, power $600 \mathrm{~mW}$, and continuous mode. The footswitch was engaged for approximately 0.5 seconds. One pulse was normally sufficient 
to establish drainage, but was repeated if no SRF appeared. Once flow of SRF was established, light pressure was maintained on the globe in order to prevent hypotony, and cryotherapy of the retinal breaks was proceeded to without delay. If bleeding from episcleral vessels obscured the view of the choroid, the sclerotomy was repeated at another site. The sclerotomy was not sutured.

SND was performed with a 5/0 ethibond spatulated needle, held in the jaws of CastroViejo forceps $2-3 \mathrm{~mm}$ from their tips. The needle was used to perforate the sclera and choroid in one smooth motion perpendicular to the scleral surface. The needle was withdrawn as the globe was simultaneously indented with the tip of a finger. The central retinal artery was observed with the indirect ophthalmoscope and sufficient pressure was achieved to close it. High pressure was maintained in order to limit bleeding from the choroidal circulation, and to facilitate passage of subretinal fluid through the small sclerotomy. After 2 minutes, the pressure was gradually reduced and the drainage site inspected by indirect ophthalmoscopy. If bleeding was observed, pressure was applied to the globe by local indentation with the cryo probe, at the drainage site, until it stopped. This manoeuvre resulted in cessation of bleeding and increased visualisation of the bleeding site.

After ALD or SND, indirect ophthalmoscopy was used to determine the result, which was graded on a four point scale as follows: (1) complete drainage of SRF, (2) partial adequate (drainage of SRF sufficient to enable the planned scleral buckling and/or intravitreal gas injection), (3) partial inadequate (insufficient drainage to allow planned scleral buckling and/or intravitreal gas injection), (4) no drainage of SRF. It was necessary to repeat the procedure in grades 3 and 4 . When repetition was necessary, the same drainage method was employed at a different site. Complications that occurred as a result of repetition were counted as complications of the original drainage procedure.

The occurrence of incarceration, retinal perforation, or subretinal haemorrhage was determined by indirect ophthalmoscopy. Haemorrhage was graded on a three point scale as follows: (1) minimal (no haemorrhage, or a spot of haemorrhage localised to the drain site and less than one disc area in size), (2) small (haemorrhage between one and four disc areas in size), (3) large (haemorrhage greater than four disc areas in size). Only small or large haemorrhages were considered to be 'clinically

Table 1 Characteristics of patients by treatment group

\begin{tabular}{llll}
\hline Characteristic & ALD & $S N D$ & p Value \\
\hline Number & 47 & 46 & \\
Average age & $55 \cdot 4$ & $55 \cdot 7$ & $0.93^{\star}$ \\
Age range & $18-88$ & $23-81$ & \\
Reoperations & $7(14 \cdot 9 \%)$ & $4(8 \cdot 7 \%)$ & $0 \cdot 55 \dagger$ \\
Macula detached & $32(68 \cdot 1 \%)$ & $32(69 \cdot 6 \%)$ & $0.95 \dagger$ \\
'Unsafe' quadrants & $5(10 \cdot 6 \%)$ & $8(17.4 \%)$ & $0 \cdot 52 \dagger$ \\
Myopia >6 dioptres & $3(6 \cdot 4 \%)$ & $4(8 \cdot 7 \%)$ & $0.97 \dagger$ \\
\hline
\end{tabular}

$\mathrm{ALD}=$ argon laser drainage; $\mathrm{SND}=$ suture needle drainage $\star$ Paired Students $t$ test. $+\chi^{2}$ with Yates' correction.
Table 2 Grade of drainage success by treatment group

\begin{tabular}{llll}
\hline & Grade of success & $\begin{array}{l}A L D \\
n(\%)\end{array}$ & $\begin{array}{l}S N D \\
n(\%)\end{array}$ \\
\hline Success & Complete & $33(70)$ & $28(61)$ \\
Failure & Partial adequate & $13(28)$ & $11(24)$ \\
& Partial inadequate & 0 & $7(15)$ \\
& No drainage & $1(2)$ & 0 \\
\hline
\end{tabular}

$\mathrm{ALD}=$ argon laser drainage; $\mathrm{SND}=$ suture needle drainage.

significant'. The grade of haemorrhage and the presence of submacular haemorrhage were determined at the time, and confirmed on the first postoperative day.

Statistical analysis was by calculation of the $\chi^{2}$ statistic with Yates's continuity correction, referred to the $\chi^{2}$ distribution with one degree of freedom. For success of drainage and size of haemorrhage, expected cell frequencies were less than Cochran's criterion. ${ }^{16}$ Cells were therefore combined in order to allow statistical analysis. When appropriate, an estimate of the difference in proportions with a particular outcome was calculated, along with its $95 \%$ confidence interval.

\section{Results}

Interim data analysis conducted after 18 months of recruitment detected a large and statistically significant difference in the incidence of clinically significant subretinal haemorrhage between the two groups. After consideration of the potential visual morbidity that may arise from this complication, a decision was made to terminate recruitment. Those already recruited numbered 93 patients, 47 in the ALD group and 46 in the SND group.

Patient characteristics were similar in each group (Table 1). There were no differences in the proportions of eyes have reoperations, or in the proportions of eyes with detached maculae. 'Safe' quadrants for drainage were defined as nasal and temporal (that is, within 1.5 clock hours of the horizontal recti). Superior and inferior quadrants were defined as 'unsafe'. The proportion of cases in which the drainage quadrant was 'unsafe' was not significantly different between the two groups. There was no difference in the proportion of eyes with myopia greater than 6 dioptres.

The success of drainage in each group is shown in Table 2 . The low expected cell frequencies precluded the use of $\chi^{2}$ testing for trend, ${ }^{16}$ so the data were combined into two categories according to whether or not the drainage needed to be repeated (Table 3 ). In four cases in the ALD group, drainage occurred before use of the laser, as a result of

Table 3 Success of drainage in each group, including an estimate of the difference in proportions and a $95 \%$ confidence interval

\begin{tabular}{lllll}
\hline $\begin{array}{l}A L D \\
n(\%)\end{array}$ & $\begin{array}{l}S N D \\
n(\%)\end{array}$ & $\begin{array}{l}\text { Difference } \\
\%\end{array}$ & $\begin{array}{l}95 \% C I \\
\%\end{array}$ & $\begin{array}{l}P \\
\text { Value }\end{array}$ \\
\hline $46(97 \cdot 9)$ & $39(84 \cdot 8)$ & -13.1 & -26.4 to 2.0 & 0.06 \\
\hline
\end{tabular}

$\mathrm{ALD}=$ argon laser drainage; $\mathrm{SND}=$ suture needle drainage.

p Values were derived by calculating the $\chi^{2}$ statistic with Yates's continuity correction and referring it to the $\chi^{2}$
distribution with one degree of freedom. 
Table 4 Complications in each group, including an estimate of the difference in proportions and a $95 \%$ confidence interval

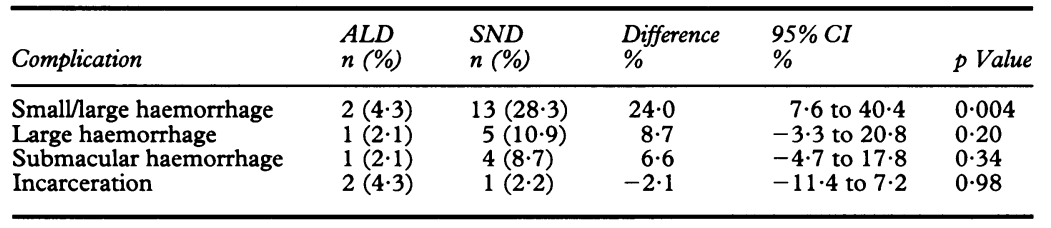

$\mathrm{ALD}=$ argon laser drainage; $\mathrm{SND}=$ suture needle drainage.

$\mathrm{p}$ Values were derived by calculating the $\chi^{2}$ statistic with Yates's continuity correction and

referring it to the $\chi^{2}$ distribution with one degree of freedom.

inadvertent perforation of the choroid by the sclerotomy blade. Two of these cases had clinically significant subretinal haemorrhages (the only haemorrhages in this group), but both had sufficient drainage to allow the planned surgery to continue. In the other two cases, repeat drainage was carried out through a further sclerotomy fashioned at a different site.

Table 4 shows the numbers and proportions of cases with each of the main complications. There was a large and statistically significant difference in the incidence of clinically significant subretinal haemorrhage between the two groups. There were $13(28 \cdot 3 \%)$ cases of small or large haemorrhage in the SND group versus two (4.3\%) in the ALD group. The lower 95\% confidence limit for the difference was $7 \cdot 6 \%$, which we consider to be a clinically significant difference. There were five large haemorrhages in the SND group, four of which became submacular, compared with one in the ALD group. This difference was not statistically significant at the $p=0.05$ level. Three haemorrhages (one large, two small) occurred in eyes in which the SND was repeated.

The distribution of eyes with subretinal haemorrhage by macular status is shown in Table 5 , and by quadrant of drainage in Table 6 . The incidence of clinically significant haemorrhage was neither higher in eyes with a detached macula, nor in eyes in which the drainage quadrant was 'unsafe'. There was one instance of clinically significant haemorrhage among the 11 reoperations, and this was in the ALD group.

There were three cases of incarceration, one in the SND group and two in the ALD group. In all three cases the incarceration was observed but no extra treatment was given. In particular the sclerotomy site was not sutured or buckled. In no case was incarceration associated with surgical failure. The case in the SND group had thin sclera and incarceration resulted from enlargement of the sclerotomy following digital pressure on the globe. No cases of retinal perforation were encountered in either group.

Table 5 Distribution of haemorrhage in eyes according to state of macula

\begin{tabular}{|c|c|c|c|c|}
\hline \multirow{2}{*}{$\begin{array}{l}\text { State of } \\
\text { macula }\end{array}$} & \multirow[b]{2}{*}{ Minimal } & \multicolumn{2}{|c|}{ Haemorrhage } & \multirow{2}{*}{$\begin{array}{l}\text { Smal } \\
\text { or } \\
\text { large }\end{array}$} \\
\hline & & Small & Large & \\
\hline Detached & 53 & 7 & 4 & $11^{\star}$ \\
\hline Attached & 25 & 2 & 2 & $4^{\star}$ \\
\hline
\end{tabular}

${ }^{\star} \mathrm{p}=0.914\left(\chi^{2}\right.$ test with Yates's correction).

\section{Discussion}

ALD was first described by Bovino et al ${ }^{10}$ and has several theoretical advantages over other methods. Retinal perforation is unlikely because there is no need to pass a sharp instrument into the subretinal space. The incidence of subretinal haemorrhage should be reduced, because of cautery of choroidal vessels by the laser. These theoretical advantages have been confirmed in practice. In a series of 200 consecutive cases there were two (1\%) instances of retinal perforation, and $11(5.5 \%)$ instances of subretinal haemorrhage, of which only one $(0.5 \%)$ was larger than $1 \mathrm{DD}$ in size ${ }^{11} \mathrm{~A}$ more recent report claimed an incidence of haemorrhage of only $1.7 \% .^{12}$ The need for a sclerotomy means that incarceration is a risk, and incidences of $2 \cdot 5 \%{ }^{11}$ and $0 \cdot 87 \%^{12}$ have been reported. The higher rate in the former study may be related to the larger L-shaped sclerotomy that was used.

SND is a simple and quick method which is easy to learn and requires no expensive equipment. A major advantage is the very small sclerotomy (the width of the needle is $340 \mu \mathrm{m}$ ) which reduces the risk of retinal or vitreous incarceration. In a series of 94 cases, successful drainage was achieved at the first attempt $76 \%$, with a final success rate of $97 \%$. There were no instances of incarceration or perforation, but an incidence of 'significant' haemorrhage of $6.4 \%$, and an overall incidence of $13.8 \%$ was reported. ${ }^{13}$

Our results indicate that drainage is more likely to be successful at the first attempt in the ALD group. The first time success rate in the ALD group was $98 \%$ compared with $85 \%$ in the SND group $(p=0 \cdot 06)$. Although there were no examples of SND which produced no SRF at all, in several cases an initial egress of fluid was followed by cessation of drainage which could not be restarted. Achieving successful drainage at the first attempt is important since it is reasonable to assume that the risk of complications increases additively for each attempt. Three of the instances of clinically significant subretinal haemorrhage in this study occurred following a repetition of drainage.

SND was associated with a higher incidence of clinically significant subretinal haemorrhage than ALD. Blood in the subretinal space may run beneath the macula, if it is detached. The blood usually clears over a period of weeks, but there may be permanent reduction in visual acuity. ${ }^{5}$ There is evidence from an experimental model of subretinal haemorrhage that photoreceptor damage can occur after only 24 hours. ${ }^{6}$ Analysis of the characteristics of each group failed to find any preoperative factor

Table 6 Distribution of haemorrhage in eyes according to quadrant of macula

\begin{tabular}{lllll}
\hline & & \multicolumn{2}{l}{ Haemorrhage } & \multicolumn{2}{l}{\begin{tabular}{l} 
Small \\
or \\
\cline { 3 - 4 } $\begin{array}{l}\text { Quadrant of } \\
\text { drainage }\end{array}$
\end{tabular}} & Minimal & Small & Large & large \\
\hline 'Safe' & 67 & 8 & 5 & $13^{\star}$ \\
'Unsafe' & 11 & 1 & 1 & $2^{\star}$ \\
\hline${ }^{\star} \mathrm{p}=0 \cdot 743$ & $\left(\chi^{2}\right.$ test with Yates's correction). &
\end{tabular}


which might account for the difference (Tables 1,5 , and 6). Raymond et al postulated that choroidal hyperaemia from recent surgery might be a factor. In our study, only one haemorrhage occurred in an eye which had undergone previous buckling surgery. One factor may be that inspection for large choroidal vessels ${ }^{7}$ is impossible using SND. It is also possible that the small size of the sclerotomy may prevent outflow of small quantities of blood along with SRF.

Induced elevation of intraocular pressure is an important step in the SND method. It must be maintained constantly until the surgeon is assured that there is no bleeding. This may present practical difficulties if the quantity of fluid drained is large - for example, if fluid from the retrohyaloid space follows subretinal fluid. In such cases an injection of intraocular air or gas may be necessary to restore volume and pressure to the globe. This must be done smoothly and rapidly, since it is our experience that a drop in pressure for even a few seconds is sufficient for significant haemorrhage to occur. In several cases, initial inspection revealed no haemorrhage at the drain site, but bleeding began following reduction of intraocular pressure after 2 minutes. A longer period of pressure elevation is then necessary, but may compromise perfusion of the optic nerve head.

There were no cases of clinically significant haemorrhage following successful use of the endolaser to perforate the choroid, but a risk of inadvertent perforation of the choroid during the dissection is inherent in this method. Both haemorrhages in the ALD group occurred following inadvertent penetration of the choroid with the blade, while fashioning the sclerotomy. The incidence of haemorrhage from this circumstance is likely to be no less than that for conventional drainage. ${ }^{1}$ It is important, however, to complete the dissection down to the level of the choroid to ensure effective drainage with the laser.

We were unable to demonstrate a significant difference in the incidence of incarceration between the two methods. Despite its occurrence in one instance, we believe that incarceration with SND is an extremely rare event, but a significant difference would only be demonstrated with a much larger sample size. The sclerotomy for ALD has to be sufficiently large to accommodate the tips of the spreading forceps and the 20 gauge endolaser probe. It is impractical with a sclerotomy smaller than $2 \mathrm{~mm}$ in length. Therefore the incidence of incarceration from ALD would be expected to be comparable with conventional drainage. ${ }^{1}$ Careful selection of the drainage site, avoidance of too rapid drainage, and a small sclerotomy all reduce the incidence and severity of incarceration to a minimum.

There were no cases of retinal perforation in our study. In SND the passage of a small needle into the subretinal space is unlikely to result in a retinal perforation, providing the drainage site has been well chosen and the globe is not indented with the needle holder. ALD is also unlikely to result in a perforation since no instrument enters the subretinal space, and any light energy reaching the detached retina is insufficient to cause a retinal hole.

In summary, the results of this trial show a higher first time success rate and a lower incidence of clinically significant haemorrhage with ALD than with SND. A difference in the rate of incarceration was not detected, although it is possible a lower incidence in the SND group may have been detected with larger numbers. We consider ALD to be the preferred method when a laser is available.

Presented at the 26th annual scientific meeting of the Retina Society, San Francisco 1993.

$S \mathrm{D}$ Schwartz was supported in part by the Ahmanson Foundation, Los Angeles, California, USA.

1 Humphrey WT, Schepens CL, Elzeneiny IL, Moura R. The release of subretinal fluid and its complications. In: Pruett RC, Regan CDJ, eds. Retina congress. East Norwalk, CT: Appleton-Century-Crofts, 1972: 383-90.

2 Hilton GF. The drainage of subretinal fluid: a randomised controlled clinical trial. Trans Am Ophthalmol Soc 1981; 79: $517-40$

3 Wilkinson CP, Bradford RH. Complications of draining subretinal fluid. Retina 1984; 4: 1-4.

4 Chignell AH, Fison LG, Davies EWG, Hartley RE, Gundry MF. Failure in retinal detachment surgery. $\mathrm{Br} f$ Ophthalmol 1973; 57: 525-30.

5 Bennett SR, Folk JC, Blodi CF, Klugman M. Factors prognostic of visual outcome in patients with subretinal haemorrhage. Am f Ophthalmol 1990; 109: 33-7.

6 Glatt H, Machemer R. Experimental subretinal haemorrhage in rabbits. Am $\mathcal{F}$ Ophthalmol 1982; 94: 762-73.

7 Freeman HM, Schepens CL. Innovations in the technique for drainage of subretinal fluid: transillumination and choroidal diathermy. Trans Am Acad Ophthalmol 1974; 8: $829-36$

8 Martin B. Controlled release of subretinal fluid. Mod Probl Ophthalmol 1975; 15: 149-53.

9 Gärtner J. Release of subretinal fluid with the aid of the microscope. Report on 100 cases. Mod Probl Ophthalmol 1975; 15: 127-33.

10 Bovino JA, Marcus DF, Nelsen PT. Argon laser choroidotomy for drainage of subretinal fluid. Arch Ophthalmol 1985; 103: 443-4.

11 Ryan EH, Arribas NP, Olk RJ, Grand MG, Boniuk I. External argon laser drainage of subretinal fluid using the endolaser probe. Retina 1991; 11: 214-8.

12 Fitzpatrick EP, Abbott D. Drainage of subretinal fluid with the argon laser. Am $\mathcal{F}$ Ophthalmol 1993; 115: 755-7.

13 Raymond GL, Lavin MJ, Dodd CL, McLeod D. Suture needle drainage of subretinal fluid. Br F Ophthalmol 1993; 77: 428-9.

14 Fleiss JL. Statistical methods for rates and proportions. 2nd ed. New York: Wiley, 1981: 41.

15 Leaver PK, Lean JS. Sclerotomy forceps. Br $\mathcal{F}$ Ophthalmol 1981; 65: 67-8.

16 Altman DG. Practical statistics for medical research. London: Chapman and Hall, 1991: 248. 